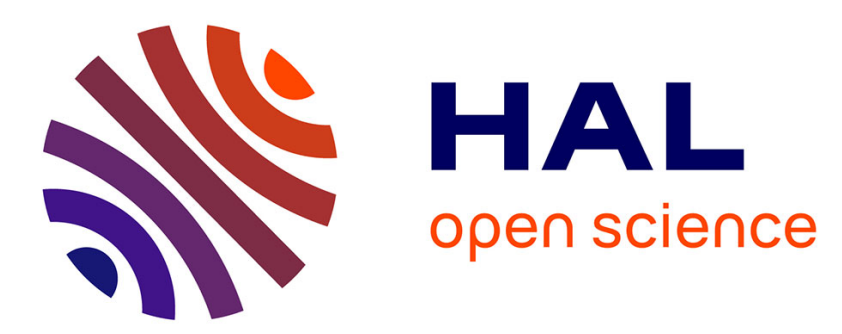

\title{
Classification of Duty Pulses Affecting Energy Storage Systems in Vehicular Applications
}

\author{
Arnaud Devie, Maxime Montaru, Serge Pelissier, Pascal Venet
}

\section{To cite this version:}

Arnaud Devie, Maxime Montaru, Serge Pelissier, Pascal Venet. Classification of Duty Pulses Affecting Energy Storage Systems in Vehicular Applications. VPPC, Sep 2010, Lille, France. pp.SS-12-6 (Actes électroniques), 10.1109/VPPC.2010.5729137 . hal-00539052

\section{HAL Id: hal-00539052 https://hal.science/hal-00539052}

Submitted on 25 Mar 2011

HAL is a multi-disciplinary open access archive for the deposit and dissemination of scientific research documents, whether they are published or not. The documents may come from teaching and research institutions in France or abroad, or from public or private research centers.
L'archive ouverte pluridisciplinaire HAL, est destinée au dépôt et à la diffusion de documents scientifiques de niveau recherche, publiés ou non, émanant des établissements d'enseignement et de recherche français ou étrangers, des laboratoires publics ou privés. 


\title{
Classification of Duty Pulses Affecting Energy Storage Systems in Vehicular Applications
}

\author{
Arnaud Devie ${ }^{* \dagger}$, Maxime Montaru* ${ }^{*}$, Serge Pelissier* and Pascal Venet ${ }^{\dagger}$ \\ *INRETS - LTE (Laboratoire Transport et Environnement) \\ 25 av Francois MITTERRAND, 69675 Bron Cedex, France \\ Email: arnaud.devie@ieee.org \\ $\dagger$ AMPERE UMR CNRS 5005 \\ Bat. Omega, Universite Lyon 1, Villeurbanne, Universite de Lyon, F.69622, Lyon, France \\ $\ddagger$ Author now works at INES CEA Laboratoire Stockage de l’Electricite \\ CEA - INES RDI, Lynx 1, 50 av du lac Leman, BP332, 73377 Le Bourget du Lac Cedex, France
}

\begin{abstract}
The study of traction batteries real-world usage in vehicular applications faces a handful of serious challenges. In this paper, we propose a new approach to evaluate real-world collected data of battery usage in EVs and HEVs. This automated method relies on $\mathrm{K}$-means clustering technique and aims at classifying duty pulses according to their current and energy distributions. We present the way data must be prepared and we discuss the results of the clustering. We believe this method enables meaningful comparison of vehicles architectures, road conditions, battery management strategies or driving behaviours. Ultimately, it allows for the elaboration of battery ageing profiles which should be more representative of real-world usage.
\end{abstract}

\section{INTRODUCTION}

The interest for electric vehicles (EV) and hybrid electric vehicles (HEV) has grown fast these past years. According to many experts in the field, the energy storage system (ESS) remains the most expensive and the most critical part among the entire electric drive. Therefore, for the sake of economic viability, it is pivotal to insure that the batteries will not fail or suffer from early weariness before the car achieves a decent mileage, 200000 kilometers being quoted quite frequently [1]. Depending upon the electrification level, traction batteries undergo very different duty profiles. The quoted goal for pure EVs batteries is 1000 cycles at $80 \%$ DoD for a $40 \mathrm{kWh}$ pack [2] whereas it is 300000 cycles at $25 \mathrm{Wh}$ or $50 \mathrm{Wh}$ per cycle for power assist HEVs batteries [3]. Between these two opposite categories of electrified vehicles comes a third one, plug-in HEVs, with mixed electrical behaviour and thus, specifically defined goal. Batteries for this latter vehicle architecture are demanded to outperform 5000 cycles at $3.4 \mathrm{kWh}$ per cycle in charge depleting mode (CD) or 300000 cycles at $50 \mathrm{Wh}$ per cycle in charge sustaining mode (CS), or any equivalent combination of both targets [4]. Even within a given electrification level category, cars from different manufacturers or driven in different environments (cold vs. mild weather, only urban vs. mixed) might exhibit totally different batteries duty profiles.

In order to make sure the installed battery pack can undergo the required mileage under realistic operating conditions, one can either perform a thorough study of batteries duty profile in real-world conditions or blindly oversize the energy storage system in order to compensate for reduced achievable mileage compared to laboratory ageing results. The later solution suffers from two major drawbacks, increased ESS weight and volume, and increased production cost. The noticeable discrepancy between real-world ageing and laboratory ageing outcomes is most likely to be due to the optimistic nature of standardized test cycles (FTP, NEDC, 10-15 Mode, ...) [5]. On the other hand, to date, real-world battery usage, related to privately owned vehicles, and to some lower extent, missionspecific vehicles, appears to be insufficiently understood and therefore, it is difficult to precisely determine the optimal battery sizing.

Pattern recognition techniques could be helpful in the process of investigating real-world battery usage. Indeed, one of the biggest challenges faced by real-world battery usage analysis lies in the fact that large amounts of collected data have to be studied. It is important to collect enough data to make sure a very broad diversity of driving situations is being addressed. Recently, a method of duty pulses classification based on fuzzy logic principles was proposed [6]. Fuzzy inference systems require the elaboration of membership functions based on the knowledge of an expert in the field (supervised classification). This appears to be a major drawback in the context of EVs battery ageing analysis because of the insufficient understanding of degradation mechanisms. However, this approach could greatly beneficiate from a learning phase such as the one performed by our non-supervised clustering approach and a hybrid approach could be envisioned.

\section{Presentation of The Developed Method}

\section{A. Data Preparation}

To address the key issue of real-world battery usage analysis, we developed a multivariate exploratory data analysis approach in order to partition the entire set of duty pulses into a chosen number of duty pulses clusters. This method is best known as $\mathrm{K}$-means clustering and we propose to apply this technique for analysis of different duty profiles of batteries. The idea behind that is to aggregate similar duty pulses into a single cluster for later examination. We have been 
collecting data about battery usage onboard a Toyota Prius II for several weeks under various road conditions: urban, extraurban and highway. The installed data logger recorded time series variables such as speed, battery voltage and current. The first step of our method is to break the current time series of each test drive down into pulses. A pulse is defined as the time series between two consecutive zero crossing abscissae. This initial sample of duty pulses is then filtered in order to discard duty pulses of short duration and low amplitude, sometimes related to noise, which tend to appear frequently but do not carry much energy and therefore are irrelevant to EV duty profile analysis. Additional details related to the selection of "relevant" pulses can be found in the discussion section. An example of transformation of time series into pulses is given in Fig. 1. This test drive is of NEDC type (full length plotted) and was performed on our dynamometer. It is of great interest to notice that the maximum amplitudes reached by the battery current are significantly lower than the values being encountered in real-world driving (less than 85 Amperes in discharge and a mere 80 Amperes in charge to be compared with 150 and 100 Amperes in real-world testing of the same vehicle). This corroborates the fact that standardized driving cycles might be of poor significance for EVs batteries duty profile analysis as stated in the introductory paragraph.

Thereafter, the term "pulses" refers to non-discarded pulses only. The second step is to express each time series duty pulse into a cumulative capacity versus current intensity representation. Fig. 2 is a time series representation of a given duty pulse and Fig. 3 is the transformed representation of the very same pulse. This step is more important than it appears because it allows for straight comparison of duty pulses and thus, this representation is best suited for the next step of our method. At this point, charge and discharge duty pulses are separated but remain treated the same way. Thus, the following steps apply for both charge and discharge cases. The third step consists of populating an array of objects with all the duty pulses. This 2-D array has as many rows as number of duty pulses in the database and has a number of columns corresponding to the number of levels chosen to quantify the current of a pulse (see Table I). Overall, 2214 charge

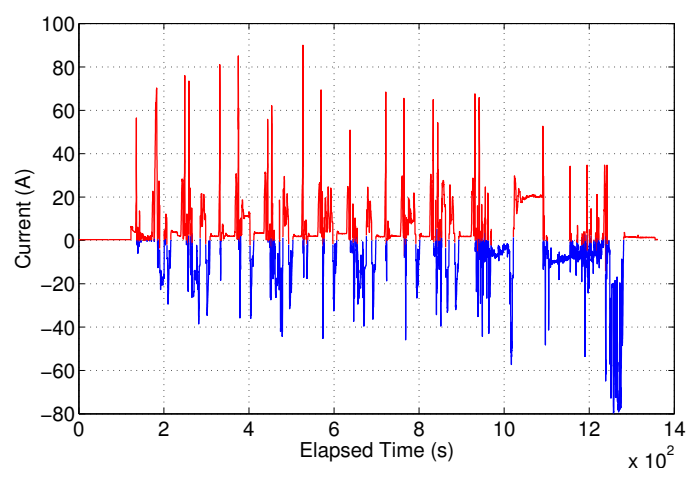

Fig. 1. Example of a test drive time series breakdown into pulses

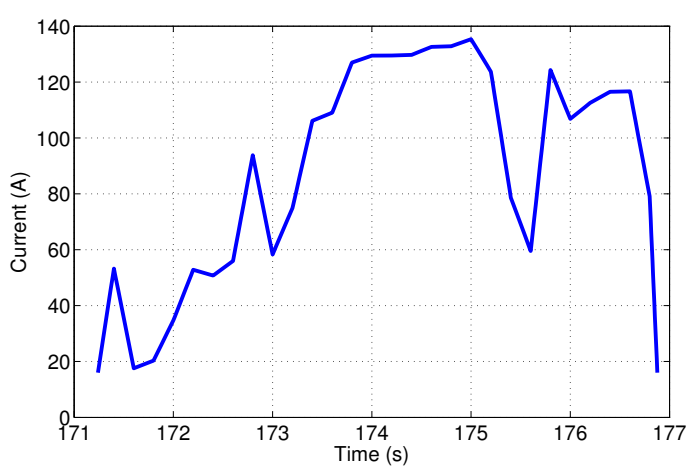

Fig. 2. Time series representation

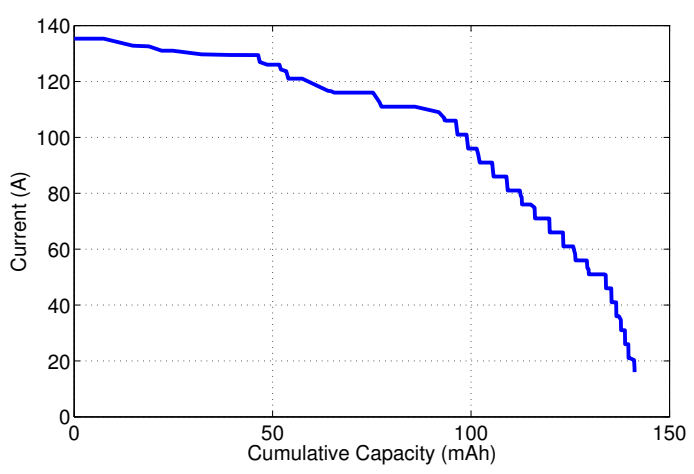

Fig. 3. Transformed representation

pulses and 3705 discharge pulses have been classified in the mixed dataset. Pulses originating from the urban dataset account for 442 charge pulses and 1124 discharge pulses. Pulses originating from the extra-urban dataset account for 549 charge pulses and 842 discharge pulses. Pulses originating from the highway dataset account for 551 charge pulses and 643 discharge pulses. For this study, $198 \mathrm{~km}$ were driven in urban area, $238 \mathrm{~km}$ were driven in extra-urban area and $348 \mathrm{~km}$ were driven on highways. Some of the test drives do not belong to any sub-dataset.

\section{B. Classification Method}

Once the array of objects is defined, a fourth step, corresponding to the K-means clustering, begins. This step starts with the selection of $\mathrm{N}$ distinct duty pulses where $\mathrm{N}$ is the desired number of clusters to be obtained. This means that

TABLE I

Charge QuANTITIES (MAH) PER CURRent LeVels (A) OF A CHARGE PULSE

\begin{tabular}{|c|c|c|c|c|c|c|}
\hline $0 . .5$ & $5 . .10$ & $10 . .15$ & $15 . .20$ & $20 . .25$ & 25.30 & $30 . .35$ \\
\hline 0 & 0.09 & 8.0 & 2.1 & 1.6 & 2.5 & 4.2 \\
\hline \hline $35 . .40$ & $40 . .45$ & $45 . .50$ & $50 . .55$ & $55 . .60$ & $60 . .65$ & $65 . .70$ \\
\hline 4.2 & 6.5 & 13.2 & 15.5 & 7.0 & 6.9 & 6.2 \\
\hline \hline $70 . .75$ & $75 . .80$ & $80 . .85$ & $85 . .90$ & $90 . .95$ & $95 . .100$ & $100 . .105$ \\
\hline 8.3 & 8.8 & 14.7 & 16.7 & 6.3 & 3.4 & 0 \\
\hline
\end{tabular}


$\mathrm{N}$ clusters are to be obtained for charge pulses and another $\mathrm{N}$ clusters are to be obtained for discharge pulses, totaling to $2 * \mathrm{~N}$ clusters for the complete analysis. The K-means clustering is known to operate in two phases. The first phase uses batch updates, where each iteration consists of reassigning points to their nearest cluster centroids, all at once, followed by recalculation of cluster centroids. The second phase uses online updates, where points are individually reassigned if doing so will reduce the sum of distances, and cluster centroids are recomputed after each reassignment [7]. This algorithm ends when no move can further increase the partitioning score (i.e. reduce the sum of distances).

\section{OUTPUTS AND RESUltS}

Once the clustering is finished, we can extract valuable results, such as the $\mathrm{N}$ clusters centroids and the relative clusters weights. The former is nothing less than the mean pulse transform of the entire cluster. It should be noted that this resulting pulse is of synthetic nature and that a time series pulse must be reconstructed for practical use. The later gives us the frequency of each type of duty pulses clusters. A representative duty profile could then be inferred from these outputs, allowing for a much simpler study of real-world usage conditions.

\section{A. Charge Pulses}

Fig. 4 reflects the variation in the results that can be obtained depending upon the operating conditions (i.e. road types). For practical purpose, the pulses are translated back onto a time-based scale. For each dataset, the relative Ah weight of each calculated class is reported in Table II. Although being of slightly shorter duration in urban traffic conditions, similar class one pulses are found throughout the whole set of data. Somehow, others classes exhibit greater variations from one traffic condition to another. The most noticeable feature variation being the increasing duration of pulses with respect to average speed (and maximum speed as well) of considered test drives (urban, extra-urban or highway). Larger

TABLE II

PROPORTIONS OF AH FOR EACH CLASS OF CHARGE PULSES

\begin{tabular}{|l|c|c|c|c|}
\hline Dataset & Class \#1 & Class \#2 & Class \#3 & Class \#4 \\
\hline Urban & $29.7 \%$ & $27.2 \%$ & $22.3 \%$ & $20.8 \%$ \\
Extra-Urban & $43.1 \%$ & $31.6 \%$ & $13.7 \%$ & $11.6 \%$ \\
Highway & $74.4 \%$ & $3.8 \%$ & $13.4 \%$ & $8.4 \%$ \\
Mixed & $55.2 \%$ & $1.7 \%$ & $30.7 \%$ & $12.4 \%$ \\
\hline
\end{tabular}

Classes definitions differ from one dataset to another

TABLE III

OCCURRENCE FREQUENCIES OF CHARGE DUTY PULSES DEPENDING ON ROAD TYPE (URBAN, EXTRA-URBAN, HIGHWAY)

\begin{tabular}{|l|c|c|c|c|}
\hline Dataset & Class \#1 & Class \#2 & Class \#3 & Class \#4 \\
\hline Urban & $89.6 \%$ & $0 \%$ & $10.0 \%$ & $0.4 \%$ \\
Extra-Urban & $71.1 \%$ & $0 \%$ & $22.1 \%$ & $6.8 \%$ \\
Highway & $88.3 \%$ & $1.1 \%$ & $6.7 \%$ & $3.9 \%$ \\
\hline
\end{tabular}

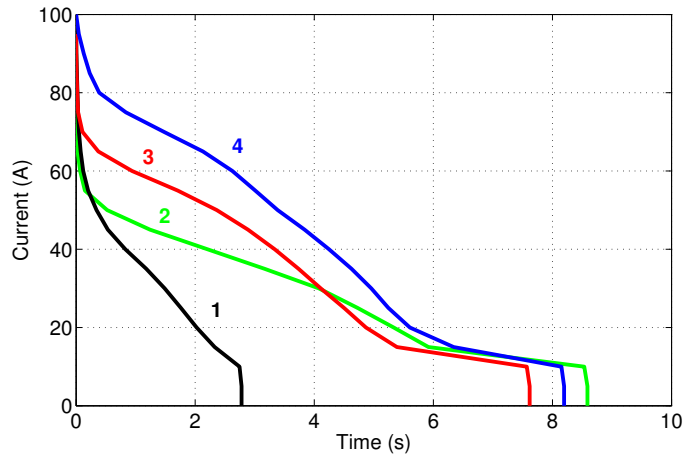

(a) Urban

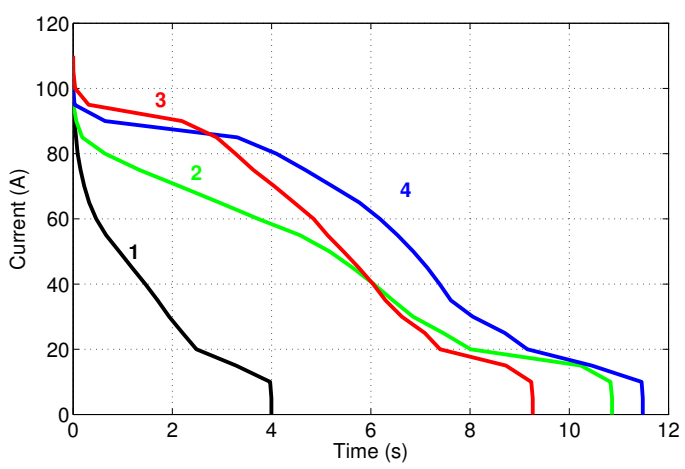

(b) Extra-Urban

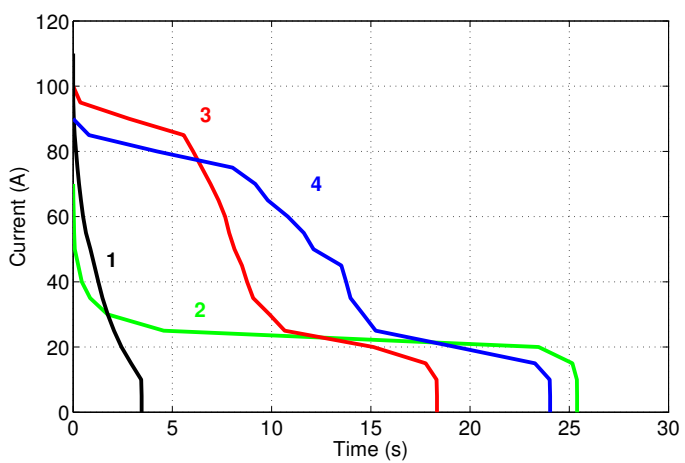

(c) Highway

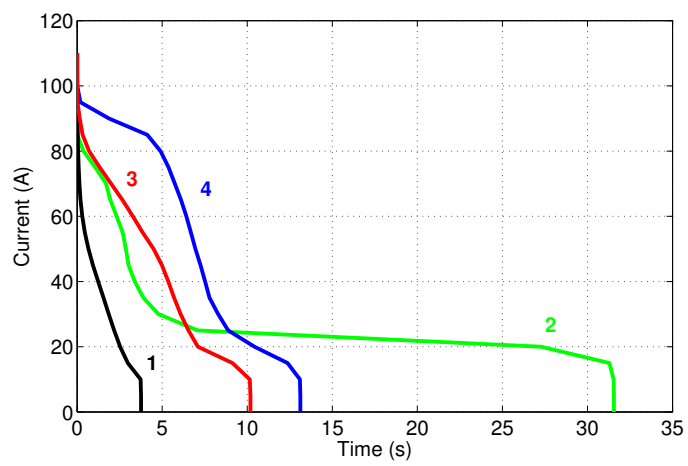

(d) Mixed

Fig. 4. Resulting typical duty pulses for $\mathrm{N}=4$ in charge case under various road conditions: (a) urban driving, (b) extra-urban driving, (c) highway driving and (d) mixed driving. 
kinetic energy harvesting potential at higher speeds certainly accounts for the observed phenomenon. Indeed, in a nonlimited recovery braking system such as the Toyota Prius one, braking from a high speed results into a larger recoverable kinetic energy than braking from an urban-compatible speed. However, when looking at Table II data, we find out that highway driving tends to trigger much less charging pulses of long duration $(74.4 \%$ of charging energy came from class one pulses). This is related to the constant speed property of highway driving, allowing for only a few complete vehicle stops (at toll booth and congestioned exit for instance). On the contrary, classes two, three and four found in urban driving account for $70.3 \%$ of charging energy involved, and their energy content (from 70 to $100 \mathrm{mAh}$ on average under 274 Volts) is typical of a complete stop from speeds ranging from 35 to $45 \mathrm{~km} / \mathrm{h}(50 \mathrm{~km} / \mathrm{h}$ being the French urban speed limit). The same goes for extra-urban driving with $56.9 \%$ of charging energy involved, and the energy content (from 140 to 180 mAh on average under 274 Volts) is typical of a $20 \mathrm{~km} / \mathrm{h}$ slow down from speeds ranging from 70 to $90 \mathrm{~km} / \mathrm{h}$. Table III displays the occurrence frequencies of the four pulses clusters generated from the mixed driving test drives dataset depending on the road types (urban, extra-urban and highway). That is, we processed the complete test drives dataset through our classification method and later examined the repartition of pulses into clusters according to their specific sub-dataset (e.g. urban). It is related to Fig. 4(d) only.

\section{B. Disharge Pulses}

Fig. 5 reflects the variation in the results that can be obtained depending upon the operating conditions (i.e. road types). Again, the pulses are translated back onto a timebased scale for practical purpose. The relative Ah weight of each calculated class can be found in Table IV. Here also, we find similar first classes in every driving situation examined. Again, the most noticeable feature variation is the increasing duration of pulses with respect to average speed of considered test drives. But in the case of discharging another explanation

TABLE IV

PROPORTIONS OF AH FOR EACH CLASS OF DISCHARGE PULSES

\begin{tabular}{|l|c|c|c|c|}
\hline Dataset & Class \#1 & Class \#2 & Class \#3 & Class \#4 \\
\hline Urban & $53.0 \%$ & $9.6 \%$ & $28.6 \%$ & $8.8 \%$ \\
Extra-Urban & $59.2 \%$ & $19.2 \%$ & $4.9 \%$ & $16.7 \%$ \\
Highway & $64.6 \%$ & $20.7 \%$ & $12.5 \%$ & $2.2 \%$ \\
Mixed & $65.2 \%$ & $16.3 \%$ & $14.6 \%$ & $3.9 \%$ \\
\hline
\end{tabular}

Classes definitions differ from one dataset to another

TABLE V

OCCURRENCE FREQUENCIES OF DISCHARGE DUTY PULSES DEPENDING ON ROAD TYPE (URBAN, EXTRA-URBAN, HIGHWAY)

\begin{tabular}{|l|c|c|c|c|}
\hline Dataset & Class \#1 & Class \#2 & Class \#3 & Class \#4 \\
\hline Urban & $92.9 \%$ & $5.4 \%$ & $1.6 \%$ & $0.1 \%$ \\
Extra-Urban & $78.7 \%$ & $15.1 \%$ & $6.1 \%$ & $0 \%$ \\
Highway & $80.1 \%$ & $10.6 \%$ & $7.9 \%$ & $1.4 \%$ \\
\hline
\end{tabular}

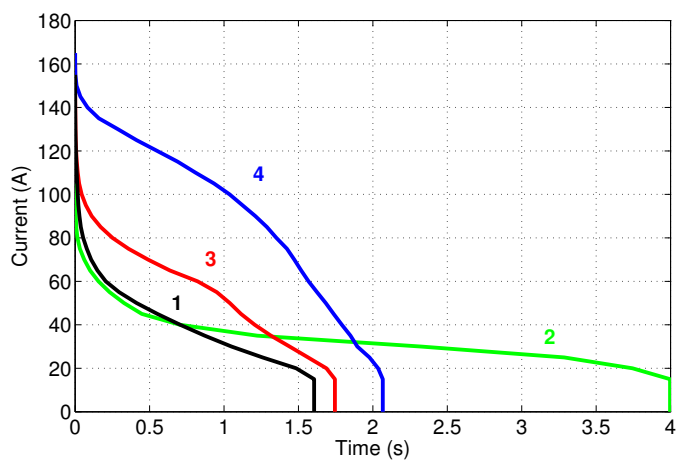

(a) Urban

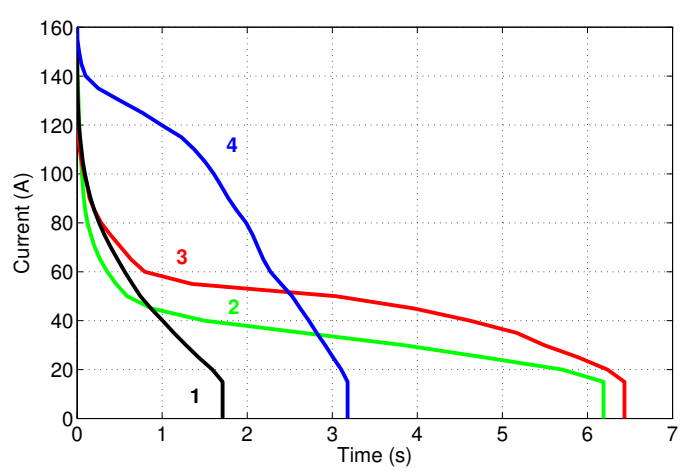

(b) Extra-Urban

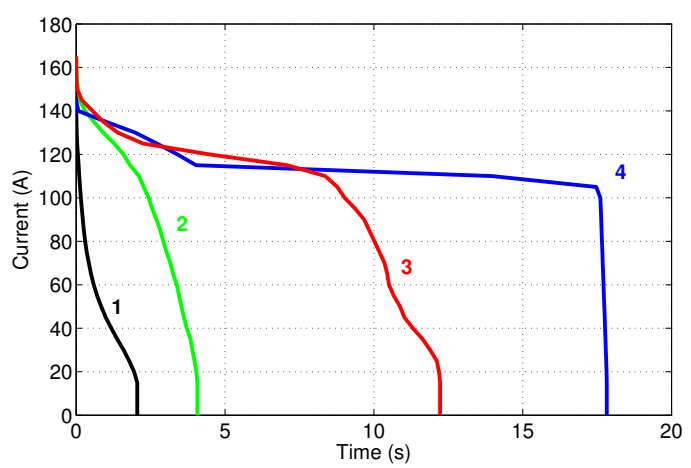

(c) Highway

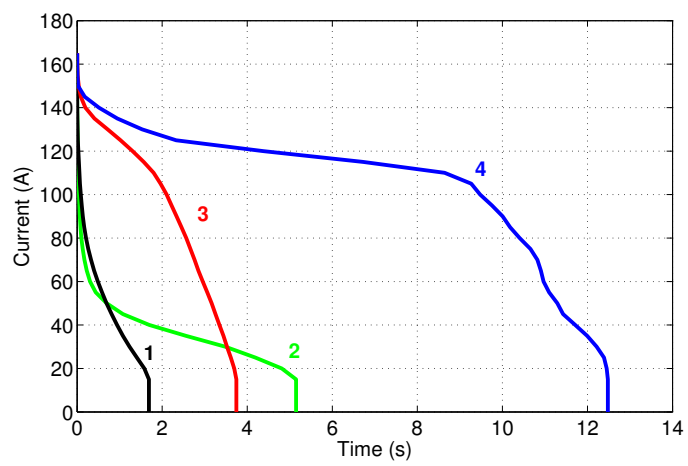

(d) Mixed

Fig. 5. Resulting typical duty pulses for $\mathrm{N}=4$ in discharge case under various road conditions: (a) urban driving, (b) extra-urban driving, (c) highway driving and (d) mixed driving. 
has to be proposed. Shorter urban pulses duration is probably due to faster driving dynamics in urban environment compared to longer accelerating events in extra-urban and highway environments. Overall, class one pulses account for a larger part of total discharging energy. Table $\mathrm{V}$ gives the occurrence frequencies of the four pulses clusters generated from the mixed driving test drives set depending on the road types (i.e. urban, extra-urban and highway). It is related to Fig. 5(d) only.

\section{DISCUSSION}

It is interesting to compare the energy content of resulting typical pulses to the $25 \mathrm{Wh}$ and $50 \mathrm{Wh}$ profiles described in [3, Section 3.9.2]. According to Table VI, under urban driving conditions, class one charging pulses carry a mere $7 \mathrm{Wh}$ on average. On the other hand, pulses belonging to others classes carry between 19 and $26 \mathrm{Wh}$ of charging energy on average with a peak value of roughly $40 \mathrm{Wh}$ for a couple of pulses. Discharging pulses carry even less energy: the mean pulse energy consumption of the first three classes establishes itself at $8 \mathrm{Wh}$ while the fourth class pulses spent $14 \mathrm{Wh}$ on average and $27 \mathrm{Wh}$ peak for some of them. As said before, similar class one pulses can be found in every cases (charging and discharging, urban, extra-urban and highway driving) and their relative contribution increases with average cruise speed. Meanwhile, energy content of others classes pulses rises with average cruise speed. Under extra-urban driving conditions, the mean energy content of non first class pulses settles at $19 \mathrm{Wh}$ in discharge and $38 \mathrm{Wh}$ in charge. Under highway driving conditions, it is close to $34 \mathrm{Wh}$ in discharge with a handful of pulses carrying between 70 and $160 \mathrm{Wh}$, and about $54 \mathrm{Wh}$ in charge. Therefore, in the case of Toyota Prius architecture, observed pulses mostly fall into the minimum power-assist $(25 \mathrm{~kW}, 25 \mathrm{Wh}$ cycle) requirements. Indeed, this $25 \mathrm{Wh}$ cycle is defined as a $25 \mathrm{Wh}$ discharge followed by a $27.8 \mathrm{Wh}$ charge. It is worth noticing that a relatively light supercapacitor based assistance in the range of 5 to $10 \mathrm{Wh}$ could handle a significant proportion of the existing duty pulses if properly managed (see first column of Tables II, IV and VI).

Such a tool enables a variety of ESS related duty profile analyses. Two approaches are possible: either performing the clustering on each test drives set (one set operated under

TABLE VI

ENERGY EQUIVALENCE OF RESULTING TYPICAL DUTY PULSES (WH)

\begin{tabular}{|l|c|c|c|c|}
\hline & \multicolumn{4}{|c|}{ Charge Pulses } \\
\hline Dataset & Class \#1 & Class \#2 & Class \#3 & Class \#4 \\
\hline Urban & 6.8 & 18.5 & 20.3 & 26.4 \\
Extra-Urban & 10.3 & 37.5 & 40.8 & 49.6 \\
Highway & 9.3 & 45.5 & 70.3 & 91.4 \\
Mixed & 9.2 & 65.6 & 33.7 & 54.6 \\
\hline & \multicolumn{5}{|c|}{ Discharge Pulses } \\
\hline Urban & 4.9 & 10.0 & 7.3 & 14.2 \\
Extra-Urban & 6.6 & 16.4 & 23.3 & 21.9 \\
Highway & 7.9 & 31.0 & 98.1 & 155.2 \\
Mixed & 9.2 & 14.4 & 33.7 & 54.6 \\
\hline
\end{tabular}

condition A and another under condition B) and comparing the shapes and levels of current of each set or performing the clustering on the joint test drives set and comparing occurrence frequencies of each cluster depending on operating conditions A or B. The former approach is illustrated by Tables II and IV, as well as Fig. 4(a)(b)(c) and Fig. 5(a)(b)(c). With this first approach, we can compare two (or more) sub-datasets according to the shapes of their resulting duty pulses. For instance, in charge case, extra-urban typical duty pulses appear to reach higher currents and carry more energy than urban typical duty pulses. The later approach is illustrated by Tables III and V, as well as Fig. 4(d) and Fig. 5(d). With this second approach, we combine two (or more) sub-datasets into a single dataset. For instance, here, we used the entire dataset (i.e. mixed dataset) which is a compilation of the three subdatasets plus some test drives that do not qualify for any specific sub-dataset. After processing this mixed dataset, we obtained four typical charge duty pulses and another four typical discharge duty pulses. These resulting eight typical duty pulses are expected to represent the whole variety of duty pulses being encountered over the general use of a Prius as a regular passenger car. We then determine the repartition of pulses coming from a given sub-dataset (e.g. urban) into resulting the clusters. If pulses from a given sub-dataset tend to be missing from a cluster whose impact on ageing is presumed larger than the others, then we can conclude that the driving conditions associated to this sub-dataset seem to be less harmful for the battery. In this study, we can notice that charge duty pulses measured under urban driving do not fall into the classes two and four (see Table III) which are anticipated to be the most harmful for the battery. They mostly fall into the first class which carries much less energy per pulse. On the other hand, we can see that most of the large energy discharge duty pulses are found among test drives operated on highway (see Table V). A typical application of this architecture variation comparison capability could be the thorough evaluation of supercapacitors assistance in terms of battery duty profile relief [8]. A designer wishing to go into further details can increase the number of clusters. To illustrate this feature, we processed the urban dataset with a target of eight clusters. Resulting typical charge pulses are given in Fig. 6. Table VII shows the relative Ah weight of each cluster. With this non-supervised clustering technique, an increase in the requested number of clusters usually results in an higher homogeneity inside clusters. This is of course a desireable feature but, on the other hand, this higher number of clusters requires more analysis time from the user, and thus a compromise must be made depending on the pursued objective. The increase in computation time is negligeable and the clustering is performed within a couple of minutes even with large datasets.

Another application of this method lies in the development of synthetic duty profiles made of a proper combination of typical duty pulses -clusters centroids- for the elaboration of more realistic time series current profiles to be used for ageing of batteries. As said before, a reconstruction step from the 


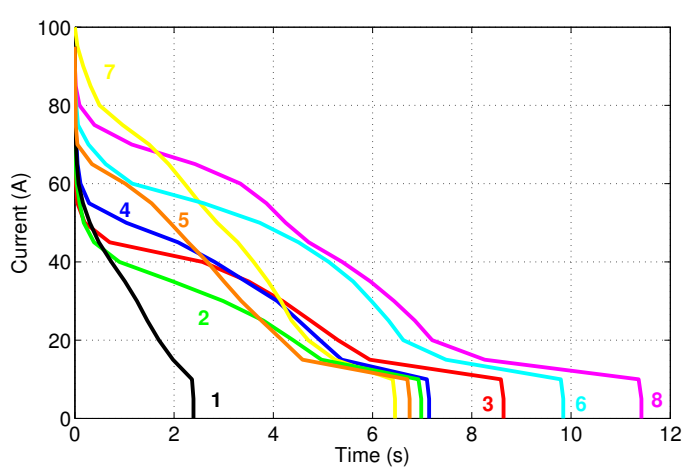

Fig. 6. Resulting typical duty pulses for $\mathrm{N}=8$ in charge case under urban driving conditions

TABLE VII

PROPORTIONS OF AH FOR EACH CLASS

\begin{tabular}{|c|c|c|c|}
\hline Class \#1 & Class \#2 & Class \#3 & Class \#4 \\
\hline $20.3 \%$ & $15.0 \%$ & $6.1 \%$ & $15.8 \%$ \\
\hline Class \#5 & Class \#6 & Class \#7 & Class \#8 \\
\hline $13.3 \%$ & $6.9 \%$ & $15.5 \%$ & $7.1 \%$ \\
\hline
\end{tabular}

resulting representation of typical duty pulses into time series current profile must be undertaken for pratical use on a battery testing system. The conversion process should be carefully designed in order to preserve pulses shapes and timings similar to real-world duty pulses. This will constitute a follow-up to this work. We expect batteries aged under these real-world inspired profiles to exhibit ageing rate and mechanisms closer to reality than usual rectangular shaped duty pulses profiles. Thus, it would be possible for automakers to accurately size the battery system for a target mileage performance. A Markov chain method will probably provide satisfactory results for the reconstruction of such real-world inspired duty profiles as in [9].

Finally, in order to tackle the frequently observed discrepancy issue between the advertised all electric range (AER) of battery electric vehicles (BEV) and plug-in hybrid electric vehicles (PHEV) and the experienced real-world range, our tool could be used in order to provide more accurate range expectancies depending upon the envisionned trip profile (i.e. urban, extra-urban and highway proportions).

Although we present results obtained with a full hybrid vehicle, this tool is meant to be used with as many vehicles as possible. The tool as is could easily accomodate data coming from other types of hybrid vehicles without significant changes. In case of BEV or PHEV duty pulses analysis, some variations are needed because of the long-lasting discharge power phases during constant speed operation that cannot be treated as "pulses". A way to deal with this issue could be to treat apart short duration pulses and long duration ones (e.g. more than a minute). Equation (1) can be modified in order to perform such a preliminary pulses sort. For instance, in this study, the criterion for selecting relevant duty pulses over irrelevant ones has been defined as follow:

$$
\Delta S o C \geq 0.15 \% \text { \& } I_{\max } \geq 40 A \& \text { Duration } \leq 45 \mathrm{~s}
$$

In this case, a pulse is said to be relevant if and only if it displaced more than $0.15 \%$ of the total battery capacity (i.e. roughly $10 \mathrm{mAh}$ or $3 \mathrm{Wh}$ energy out of the $6.5 \mathrm{Ah}-1.8 \mathrm{kWh}$ Prius II battery), reached a current amplitude greater than 40 Amperes and lasted less than 45 seconds. The two former parameters are useful for reducing the number of low energy pulses; a treshold decrease would translate into an increase of the short pulses population and would therefore partially shade the visibility of larger pulses. For this study we wanted to focus on larger pulses for their anticipated effects on battery ageing. The latter parameter is used to prevent the "pollution" of results by long lasting discharging and charging episodes artificially provoked during vehicle testing by means of manual battery SoC alteration. A full performance BEV operated in varied driving environments would most likely need a larger number of clusters than used for the Prius in order to limit the loss of information resulting from our simplifying tool. This is because the battery pack would be used in a broader spectrum of situations and for a larger portion of time over the same trip.

\section{CONCLUSION}

We propose a multivariate exploratory data analysis approach to analyze real-world usage of batteries in EVs and HEVs. The method has been applied to a variety of test drives of a Toyota Prius II and typical duty pulses have been identified for each driving conditions (i.e. urban, extra-urban, highway and mixed). We believe that electric drive architecture variations can be compared in a meaningful manner with the help of this developed tool allowing for a much simpler evaluation of one solution over another in terms of ESS duty profile. We also promote this tool for elaborating real-world inspired battery ageing profiles instead of non-realistic existing profiles.

\section{ACKNOWLEDGMENT}

This work was supported by Region Rhone-Alpes and ADEME.

\section{REFERENCES}

[1] PNGV, Battery Test Manual, PNGV, October 2003

[2] USABC, Goals for Advanced Batteries for EVs, USABC, 2002.

[3] - FreedomCAR Power Assist Goals, USABC, 2002.

[4] —, Plug-in HEV Battery Goals, USABC, 2008.

[5] J. Groot, "Battery benchmarking and cyclelife test methods," in Transport Research Arena Europe 2010, Brussels, 2010.

[6] B. Y. Liaw and M. Dubarry, "From driving cycle analysis to understanding battery performance in real-life electric hybrid vehicle operation," Journal of Power Sources, vol. 174, no. 1, pp. 76 - 88, 2007, hybrid Electric Vehicles.

[7] Statistics Toolbox, Kmeans function help file, The MathWorks, Inc., 2009.

[8] A. Allegre, A. Bouscayrol, and R. Trigui, "Influence of control strategies on battery/supercapacitor hybrid energy storage systems for traction applications," in Vehicle Power and Propulsion Conference, 2009. VPPC '09. IEEE, sept. 2009, pp. $213-220$.

[9] J. Groot, "Statistic method for extraction of synthetic load cycles for cycle life tests of hev li-ion batteries," in EVS24 International Battery, Hybrid and Fuel Cell Electric Vehicle Symposium, 2009. 